\title{
Defective Number Sense or Impaired Access? Differential Impairments in Different Subgroups of Children With Mathematics Difficulties
}

\author{
Terry T.-Y. Wong, PhD', Connie S.-H. Ho, PhD², \\ and Joey Tang, PhD ${ }^{3}$
}

\begin{abstract}
Developmental dyscalculia (DD) is a specific learning disability in mathematics that affects around $6 \%$ of the population. Currently, the core deficit of DD remains unknown. While the number sense deficit hypothesis suggests that the core deficit of DD lies in the inability to represent nonsymbolic numerosity, the access deficit hypothesis suggests that the origin of this disability lies in the inability to associate numbers with the underlying magnitude representation. The present study compared the performance of DDs with their low-achieving (LA) and normally achieving peers in nonsymbolic numerosity processing and number-magnitude mapping over I year (from kindergarten to Ist grade). The results demonstrated differential impairments in different subgroups of children with mathematics difficulties. While DDs showed deficits in both nonsymbolic numerosity processing and number-magnitude mapping, LAs showed deficit only in the number-magnitude mapping. Furthermore, the deficit in number-magnitude mapping among the DD group was partially explained by their number sense deficit. The number sense deficit hypothesis is supported. Theoretical and practical implications are discussed.
\end{abstract}

\section{Keywords}

dyscalculia, mathematics

In the modern numerate society, having adequate numeracy skills not only enables people to gain more education and better career prospects but also protects them from having poor physical and mental health (e.g., at a lower risk of having depression; Parsons \& Bynner, 2005). However, a small portion of the population experiences some specific, persistent difficulties in learning mathematics. This population is known as having developmental dyscalculia (DD), and they account for around $6 \%$ to $7 \%$ of the school-age population in Western societies (Butterworth, 2005; Shalev, 2007).

Increasing numbers of studies have been done to find out the core underlying cognitive deficits among dyscalculics (e.g., Geary, Hoard, Byrd-Craven, \& Nugent, 2007; Landerl, 2013; Mazzocco, Feigenson, \& Halberda, 2011; Rousselle \& Noël, 2007). Two major hypotheses have been put forward, and they are the number sense deficit hypothesis (Wilson \& Dehaene, 2007) and the access deficit hypothesis (Rousselle \& Noël, 2007). While the former hypothesis proposes that dyscalculia originates from a deficit in processing nonsymbolic numerosity, the latter hypothesis suggests that the core deficit lies in the inefficient access of magnitude representation from the number symbols. Details of the two hypotheses are discussed in the following.

\section{Innate Number Sense and the Number Sense} Deficit Hypothesis

The Approximate Number System (ANS) has been proposed to be one of the innate systems that allows humans to represent numerosity (Feigenson, Dehaene, \& Spelke, 2004). This system allows humans to represent numerosity in an approximate manner. People cannot distinguish between two numerosities unless the ratio between them is large enough. The ANS has been suggested to be encoding numerosities as analog magnitudes along a mental number line, which is compressed on a logarithmic scale (Dehaene, Piazza, Pinel, \& Cohen, 2003; Piazza, Izard, Pinel, Le Bihan, \& Dehaene, 2004). It is therefore more difficult to

\footnotetext{
'The Hong Kong Institute of Education

${ }^{2}$ The University of Hong Kong

${ }^{3}$ Society for the Promotion of Hospice Care, Hong Kong

Corresponding Author:

Terry, T.-Y. Wong, Room 24, 2/F, DI, The Hong Kong Institute of Education, 10 Lo Ping Road, Tai Po, New Territories, Hong Kong 852, Hong Kong.

Email: terrytywong@gmail.com
} 
discriminate between large numerosity pairs than small ones, given that they are of the same absolute difference. The finest ratio of two numerosities that can be reliably distinguished is called the number acuity, captured by the Weber Fraction $(w)$. Using the nonsymbolic comparison task, in which participants are given two arrays of dots and objects and are asked to pick the more numerous array, previous findings suggest that our number acuity improves with age (Halberda, Ly, Wilmer, Naiman, \& Germine, 2012; Piazza et al., 2010)

This innately equipped ANS was suggested to be the basis of our symbolic math skills (Dehaene, 2001). The first behavioral evidence of this came from Halberda, Mazzocco, and Feigenson (2008), in which they found that the number acuity of 14-year-old adolescents retrospectively correlated with their previous mathematics achievement. Although the relation between ANS and mathematics is not without controversy (De Smedt, Noël, Gilmore, \& Ansari, 2013), a recent meta-analysis, which included 36 samples, revealed a moderate but significant correlation between ANS and mathematics performance (Chen \& $\mathrm{Li}, 2014)$. The authors also suggested that the small sample size in some of the studies might have accounted for the null findings in those studies. Furthermore, recent studies found that the number acuity of 4-year-old children was predictive of their mathematics abilities after half a year, even after controlling for their previous mathematics abilities (Libertus, Feigenson, \& Halberda, 2013), and numerical preference (a measure of infants' number acuity) at 6 months predicted math ability at 3.5 years old after controlling for general intelligence (Starr, Libertus, \& Brannon, 2013). Both findings suggest that the ANS may serve as the foundation of our symbolic math skills.

With all the above evidence demonstrating a significant relation between number sense and our symbolic mathematics skills, it was suggested that the deficit in this number sense may be a major underlying cause of dyscalculia (Wilson \& Dehaene, 2007; also see Butterworth, 2005, for a similar hypothesis on exact numerosity processing). Children who have a more fuzzy number sense, which prevents them from clearly distinguishing numerosities, may turn out to have difficulties in learning symbolic mathematics. Several pieces of evidence support the hypothesis by showing that dyscalculics tend to have lower acuity in numerosity representation (i.e., a higher average $w$ score; Mazzocco et al., 2011; Piazza et al., 2010). However, other studies did not observe such a relation (i.e., De Smedt \& Gilmore, 2011; Iuculano, Tang, Hall, \& Butterworth, 2008; Landerl \& Kölle, 2009; Rousselle \& Noël, 2007).

\section{The Access Deficit Hypothesis}

Although the ANS may serve as the foundation of our symbolic math skills, it does not seem to be the only required condition. Numbers have to be mapped onto the underlying representation of magnitude in order to acquire their meaning (Dehaene, 2005). Even with an intact ANS, people may still fail in learning mathematics if there are problems in mapping the number symbols onto the underlying magnitude representation. Some researchers proposed that a major domainspecific deficit of dyscalculia originated from an inability to relate number symbols to the underlying magnitude representation instead of an inability to represent numerosity per se (Rousselle \& Noël, 2007). In other words, the dyscalculics have intact number sense, but they have difficulties in processing symbolic numbers due to the ineffective linkage between the number symbols and the underlying numerosity representation. If this is the case, dyscalculics should be outperformed by their normally achieving (NA) peers in symbolic numerical tasks but not in nonsymbolic ones.

The access deficit hypothesis has received support from various studies. In the initial study in which the hypothesis was proposed, second graders with mathematics difficulties were compared with a group of chronological age controls on both symbolic and nonsymbolic comparison tasks. While there was a clear difference in their performance in the symbolic task, with the control group significantly outperforming the mathematics difficulty group, the performance of the two groups were highly similar in the nonsymbolic task (Rousselle \& Noël, 2007), suggesting that their difficulties were limited to symbolic numerical processing. De Smedt and Gilmore (2011) further examined whether the access deficit also applied to a younger age group (i.e., first graders). By using multiple measures (symbolic and nonsymbolic comparison, symbolic and nonsymbolic addition), they have shown that children with mathematics learning disabilities showed worse performance in symbolic, but not nonsymbolic, numerical measures. The findings have provided further support to the access deficit hypothesis.

\section{Controversies of the Two Hypotheses}

Existing findings regarding the above two hypotheses seem to be inconclusive. Two reasons may have contributed to this controversy. First, the items used in the nonsymbolic comparison task differed across studies. For example, while the nonsymbolic comparison tasks in some studies involved relatively large ratios (e.g., ratios range from 1.5 to 2 in Rousselle \& Noël, 2007), others involved some smaller ratios (e.g., ratios range from 1.06 to 1.33 in Piazza et al., 2010). Given a $w$ fraction of about 0.25 to 0.34 between 5 to 10 years old (Piazza et al., 2010), the nonsymbolic comparison task should involve a considerable number of items within the range of 1.25 to 1.34 (roughly equivalent to a ratio of $4: 5$ to $3: 4$ ) in order to detect individual differences. We addressed this issue in our current study by using a wider range of ratios in the nonsymbolic comparison task in order to provide a more sensitive measure of children's number acuity. 
Second, the use of different criteria in defining dyscalculia among different studies may also lead to different results. Most of the studies on dyscalculia used standardized mathematics achievement tests for identifying children with dyscalculia, but the cutoff values used in different studies varied a lot, ranging from 5th percentile to 45th percentile (see Murphy, Mazzocco, Hanich, \& Early, 2007, for a summary). The use of different criteria makes it difficult for researchers to ensure that they are studying the same target population. In fact, the use of different criteria was found to affect the cognitive profiles of the identified samples (Murphy et al., 2007). The same logic applies to the studies that compared the number sense deficit hypothesis and the access deficit hypothesis. While a cutoff point of 15 th percentile was used in the studies by Rousselle and Noël (2007) and De Smedt and Gilmore (2011), which was already more stringent than many other studies on dyscalculia (e.g., Geary, Hamson, \& Hoard, 2000; Hanich, Jordan, Kaplan, \& Dick, 2001), still more stringent criteria were used in some studies (e.g., 10th percentile for Mazzocco et al., 2011; performance below $2 S D$ in one of the two quotients in Piazza et al., 2010). In fact, the use of more stringent criteria resulted in prevalence rates that are much closer to the proposed prevalence rate of $6 \%$ to $7 \%$ (Butterworth, 2005; Shalev, 2007). While the former two studies failed to find any significant differences between dyscalculics and NA children in their performance in nonsymbolic comparison tasks, significant differences were observed in the latter two studies. The above comparison seems to suggest that deficit in nonsymbolic numerosity processing are confined to the population with more severe difficulties in mathematics. The current study examined this possibility by comparing the nonsymbolic numerosity processing among children with dyscalculia (bottom 10th percentile in the standardized achievement test) with their low-achieving (LA; between 11th and 25th percentile in the standardized achievement test) and NA peers.

Even though the research findings seem to support the access deficit hypothesis, we are far from conclusive on whether dyscalculics have deficits in accessing numerosity representation from number symbols. This is primarily because a symbolic comparison, in which participants decide which of the two presented Arabic numerals is larger in magnitude, is typically used for measuring the access of the underlying magnitude representation from the number symbols. While the task itself does not involve any nonsymbolic magnitude, it seems to be measuring symbolic number processing instead of the access of magnitude representation from number symbols. While it has been traditionally assumed that numbers were mapped onto the magnitude representation to acquire their meanings (e.g., Dehaene, 2005), other mechanisms remain possible. For example, it was proposed that the system of natural numbers was developed first and was associated with the innate number sense only at a later time point
(Carey, 2004; Noël \& Rousselle, 2011). In other words, the substandard performance in the symbolic comparison task among dyscalculics does not necessarily mean that they have deficits in accessing magnitude representation from the number symbols. To more clearly assess the validity of the access deficit hypothesis, the measures should tap more directly onto the number-magnitude mapping process (i.e., they should involve both symbolic numbers and nonsymbolic magnitudes).

Two daily-life numerical skills, namely object counting and estimation, have been proposed to reflect how well the number symbols are mapped onto our underlying representation of numerical magnitude (Wong, Ho, \& Tang, 2015). Object counting is undoubtedly the first systematic procedure that associates number symbols with our underlying representation of magnitude. It allows us to acquire the meaning of these number symbols, which are the foundation of all other kinds of mathematical skills. It also provides a way for us to do the most fundamental form of arithmetic. Because of this, it is not surprising that children's counting skills are predictive of their later mathematics achievement (e.g., Reigosa-Crespo et al., 2012). It is also reasonable to suggest that those who fail to count would encounter difficulties in learning mathematics. In fact, some research findings suggest that dyscalculics counted slower than their same-age peers (Landerl, Bevan, $\&$ Butterworth, 2004) and that children who were identified as slow counters showed worse performance in arithmetic, compared with medium and fast counters (Reeve, Reynolds, Humberstone, \& Butterworth, 2012).

Estimation, or pure numerical estimation, refers to a process that has a goal of approximating some quantitative value and with numbers as inputs, outputs, or both (Booth \& Siegler, 2006). Real-world knowledge is not relevant during pure numerical estimation. Examples of estimation include estimating the number of people in a shop as well as estimating the position of 37 in a 0 to 100 number line. As the process of estimation involves translating a magnitude to a number, or vice versa, it has been proposed to reflect the mapping between number symbols and the underlying magnitude representation (Wong et al., 2015). The strong relation between children's performance in a number line task and arithmetic achievement further confirms this possibility (Fuchs et al., 2010; Siegler \& Booth, 2004). On top of that, children with dyscalculia were also found to make significantly more errors in the number line estimation task (Geary, Hoard, Nugent, \& Byrd-Craven, 2008).

\section{The Relation Between Number Sense and Number-Magnitude Mapping}

On top of the issues concerning the two hypotheses alone, it is also questioned how the two processes described by the two hypotheses are related. More specifically, what is 
the relation between our number sense and access of magnitude representation from number symbols? The framework proposed by Wong et al. (2015) may provide some insights on this issue. In that study, it was found that the effect of number sense on children's mathematics achievement was fully mediated by number-magnitude mapping (measured by object counting and estimation). This finding suggests that if children are weak in their number sense, they may also encounter problems in accessing magnitude representation from the number symbols, and which may therefore lead to difficulties in learning mathematics. For example, a child having difficulty in distinguishing numerosities of four and five may not be able to precisely map the Arabic numeral "4" onto its underlying representation. In other words, the number sense deficit may lead to access deficit. However, it is also possible that some children may have impaired access alone, without any difficulties in the number sense.

\section{The Current Study}

The primary aim of the present study was to examine the relevance of the number sense deficit hypothesis and the access deficit hypothesis in explaining the difficulties experienced by children with dyscalculia. A dyscalculic group was compared with a LA group and a NA control group on various tasks accessing their number sense and access of magnitude representation from number symbols (counting, estimation, symbolic comparison). A deficit in number sense, along with a deficit in accessing magnitude representation from number symbols, was expected in the dyscalculic group. A longitudinal design was employed so that early cognitive predictors of dyscalculia could be identified.

\section{Method}

\section{Participants}

A total of 211 (110 boys and 101 girls) Chinese children were originally recruited from 17 kindergartens in Hong Kong for the present study. These children were tested four times in 3 years (end of kindergarten, middle of first grade, end of first grade, and end of second grade). The final sample in Wave 4 consisted of 141 second-grade children (73 boys and 69 girls, mean age $=8.08$ years, $S D=0.33$ years). Participants with IQs less than 80 or attention-deficit/hyperactivity disorder were excluded from the sample. Among the 141 children remaining in the final sample, we identified a group of LA children and another group of children with DD. While the DD group scored below the 10th percentile in a standardized achievement test on mathematics, the Learning and Achievement Measurement Kit 2.0 (LAMK 2.0; Hong Kong Education Bureau, 2008), the LA group scored between the 11th and 25 th percentile on the same achievement test. Using the above criterion, 13 children $(9.2 \%)$ were identified as having DD, while 20 children $(14.2 \%)$ were identified as LA. The rest of the sample $(n=108,76.6 \%)$ was considered NA.

\section{Measures}

All the number-related cognitive measures were computerized measures, and the achievement and control (IQ and verbal memory) measures were conducted in paper-andpencil or verbal format.

\section{Number-related cognitive measures}

Number sense (ANS). The number sense of the participants was assessed using the nonsymbolic comparison task adopted from Piazza et al.'s (2004) study. The participants were presented with two arrays of dots with varying dot sizes (ranging from a diameter of 6 pixels to a diameter of 58 pixels), and they had to decide which array contained more dots without counting (on average, participants took less than $2 \mathrm{~s}$ for each trial, suggesting that they did not engage in counting while performing the task). One of the arrays always contained 16 dots, while the other array consisted of 10 to 22 dots. The ratios between the numerosity of the dots ranged from 1.06 to 1.60 . While the ratios greater than 1.2 were considered large ratios (i.e., 1.23, 1.25, 1.33, 1.38 , and 1.6), those less than 1.2 were considered small ratios (i.e., 1.06, 1.07, 1.13, 1.14, and 1.19). In half of the trials, the average dot size was directly proportional to the numerosity (congruent trials); while in the other half of the trials, the relation between average dot size and numerosity was an inverse one (incongruent trials). To familiarize the participants with the task, five practice trials were given before 50 experimental trials. Both raw scores and $w$ were used in the analyses.

Object counting. A dot-number matching task similar to the one in Dyscalculia Screener (Butterworth, 2003) was administered in the current study to measure the participants' object counting skills. The participants saw an array of dots on one side of the screen, and they were asked to count the number of dots to decide whether the numerosity of the array matched the Arabic numeral on the other side of the screen. A time limit of $8 \mathrm{~s}$ was imposed on the task, and the number involved in the tasks ranged from 1 to 9. Participants were given 4 practice trials before they attempted the 36 experimental trials. The counting performance of the participants was reflected by both accuracy and reaction time.

Estimation. The participants' estimation skills were assessed using the number line estimation task adopted from Siegler and Booth's (2004) study. In this task, participants were shown a number line of 0 on the left end and 100 
on the right end. They also saw an Arabic numeral above the number line, and they had to move the cursor to the position where the Arabic numeral should be located. A time limit of $30 \mathrm{~s}$ was set in each item. There were 3 practice trials followed by 24 experimental trials. Both linearity $\left(\mathrm{R}_{\text {lin }}^{2}\right.$, assessing the fit of the participants' responses against the best-fit linear function) and percentage absolute error (PAE) were used in the analyses.

Symbolic comparison. A symbolic comparison task was used to measure participants' symbolic number processing skills. Participants were shown two single-digit Arabic numerals, and they were asked to judge which Arabic numeral represented had a larger numerical value and to respond by key pressing. There were 4 practice trials, followed by 36 experimental trials. Both accuracy and average reaction time were used as the indices of participants' symbolic number-processing skills.

Mathematics achievement. The LAMK 2.0 (Hong Kong Education Bureau, 2008) on mathematics was used to assess participants' achievement in mathematics. The LAMK 2.0 is a standardized assessment tool developed by the local education bureau, with the aim of identifying children who need remediation in their learning. The version for second graders was conducted in the last wave of testing. This version consists of 33 items in total, mostly tapping children's arithmetic skills, but also includes items on shape and space, measures, and statistics. Participants were given $45 \mathrm{~min}$ for this task. Based on the local norm calibrated by the Rasch model, the raw scores of the participants were converted into grade-level scores (ranged from 11 to 1,000, with a score of 300 or greater suggesting a grade-appropriate performance), which were taken as the indicator of participants' mathematics achievement.

\section{Control measures}

Nonverbal intelligence. The short form of Raven's Standard Progressive Matrices (Raven, 1956) was used to assess participants' nonverbal intelligence. Each item consisted of a visual pattern with a missing piece, and the participants had to identify the correct piece, out of six options, that completed the pattern. Each correctly answered item yielded one mark. The short form consisted of set A to set $\mathrm{C}$, with a total of 36 items. Raw scores were converted into scaled scores based on local norms.

Verbal memory. The syllable recall task was used to assess participants' verbal memory capacity. The participants were verbally presented a series of Chinese syllables, one per second, and they were to repeat the syllables in the correct order after listening. There was a practice item, followed by 15 experimental items in five difficulty levels. There were three syllables in the first level, and each ascending level has one more syllable compared to the previous level. Each correctly recalled syllable and correct order yielded one mark.

\section{Procedures}

Invitation letters were first sent to 96 kindergartens in Hong Kong to invite their participation. Seventeen kindergartens agreed to participate, and 211 parental consents were received. Testing was conducted in the kindergartens in the first wave of assessment, while the follow-up assessments were conducted at the participants' homes. While the cognitive tasks (i.e., dot-number matching, number line, symbolic and nonsymbolic comparison) were administered in the first three waves of assessment, the control measures were conducted in only one of the waves (verbal memory in Time 1 and nonverbal intelligence in Time 2), and the achievement measure was conducted only in Time 4. Each assessment session lasted for around 1.5 hours (including other tasks that were not reported in the present study). All assessments were conducted by trained psychology undergraduates or the first author. Participants were given small souvenirs in return for their effort in the assessments.

\section{Results}

Before conducting the analysis, all the outliers, that is, data points that were $3 S D$ s beyond the group means, were deleted (a total of 26 data points were deleted, 22 from the NA group and 4 from the LA group, none from the DD group). First, we compared the three groups in terms of their age, mathematics achievement, nonverbal intelligence, and verbal memory using ANOVA, with group identity being the independent variable. Significant comparisons were followed up by post hoc analyses with Bonferroni adjustment. For the comparisons in which unequal variances were found, the Welch $F$ were used, and the GamesHowell test were used for the post hoc analyses.

The results of the ANOVA suggested that the three groups were of similar age across all the assessment time points, $F(2,138)<.6, p \mathrm{~s}>.5$. As expected, the groups significantly differed in terms of their mathematics achievement, Welch $F(2,31.691)=230.959, p<.001, \mathrm{y}_{\mathrm{p}}{ }^{2}=.547$. The post hoc analysis suggested that the NA group performed significantly better than the LA group $(p<.001)$, which also significantly outperformed the DD group $(p<$ $.001)$. The groups also significantly differed in terms of their nonverbal intelligence, Welch $F(2,28.534)=24.079$, $p<.001, \mathrm{y}_{\mathrm{p}}{ }^{2}=.200$, and verbal memory, $F(2,24.413)=$ $8.229, p=.002, \mathrm{y}_{\mathrm{p}}^{2}=.116$. For nonverbal intelligence, the NA group significantly outperformed the LA and the DD group $(p \mathrm{~s}<.001)$, while the latter two groups did not differ significantly $(p=.583)$. For verbal memory, the NA group significantly outperformed the LA group $(p=.004)$ and 
Table I. Means, Standard Deviations, and the Results of ANOVA for Mathematics Achievement and Control Measures.

\begin{tabular}{|c|c|c|c|c|c|c|}
\hline \multirow[b]{3}{*}{ Variable } & \multicolumn{3}{|c|}{ Group mean } & \multicolumn{3}{|c|}{ ANOVA } \\
\hline & \multirow[b]{2}{*}{$\mathrm{DD}(n=13)$} & \multirow[b]{2}{*}{$\operatorname{LA}(n=20)$} & \multirow[b]{2}{*}{$\mathrm{NA}(n=108)$} & \multicolumn{2}{|l|}{ Math main } & \multirow[b]{2}{*}{ Post hoc } \\
\hline & & & & Welch $F$ & Partial $\eta^{2}$ & \\
\hline LAMK 2.0 & $279.154(38.817)$ & $374.500(31.296)$ & 692.361 (I57.835) & $F(2,32.691)=230.959 * * *$ & .547 & $N A>L A>D D$ \\
\hline $\begin{array}{l}\text { Nonverbal } \\
\text { intelligence }\end{array}$ & $100.23 \mid(8.408)$ & $103.250(8.577)$ & II $5.009(11.821)$ & $F(2,28.534)=24.079 * * *$ & .200 & $N A>L A=D D$ \\
\hline Verbal memory & 71.077 (25.607) & $77.300(14.477)$ & $90.028(18.07 I)$ & $F(2,24.413)=8.229 * *$ & .116 & $N A>L A ; N A>D D^{+}$ \\
\hline
\end{tabular}

Note. DD = developmental dyscalculia; LA = low-achieving; NA = normally achieving; LAMK $2.0=$ Learning and Achievement Measurement Kit 2.0. ${ }^{+} p<.07 . *^{*} p<.01$. *** $p<.001$.

marginally significantly outperformed the DD group $(p=.054)$. The latter two groups did not differ in their verbal memory ( $p=.710$; see Table 1 for details).

The groups' performances on various number sense and number-magnitude mapping tasks were analyzed using 3 (Group) $\times 3$ (Time) repeated-measures ANCOVA (except for the analyses on nonsymbolic comparison accuracy, in which a factor of ratio was further introduced), with nonverbal intelligence and verbal memory being the covariates. The Greenhouse-Geisser correction was used in the analyses, which yielded significant sphericity (i.e., all except nonsymbolic comparison accuracy and $w$ and dot-number matching accuracy). On the other hand, for the comparisons with unequal variances (i.e., ratio of largest group variance to smallest group variance $>2$ ), the critical alpha level would be adjusted to a value of .01 (Tabachnick \& Fidell, 2006). This applied to the analyses on nonsymbolic comparison $w$, number line, and symbolic comparison accuracy. Significant differences were further examined by post hoc analyses with Bonferroni adjustment. The group performances in these tasks are shown in Figures $1 \mathrm{~A}$ to $1 \mathrm{H}$.

\section{Nonsymbolic Comparison}

We analyzed the data of the nonsymbolic comparison task in terms of both accuracy and $w$. The $w$ of the participants were calculated using the psychophysical modeling technique commonly used in the literature (Halberda et al., 2008; Piazza et al., 2010; Starr et al., 2013): error rate $=\frac{1}{2} \operatorname{erfc}\left(\frac{\mathrm{n}_{1}-n_{2}}{\sqrt{2} \mathrm{w} \sqrt{\mathrm{n}_{1}^{2}+\mathrm{n}_{2}^{2}}}\right)$, where $\mathrm{n}_{1}$ and $\mathrm{n}_{2}$ are the numerosities of the larger and smaller set, respectively, $w$ is the Weber fraction, and erfc is the complementary error function.

For accuracy, the 3 (Group) $\times 3$ (Time) $\times 2$ (Ratio) repeated-measures ANCOVA suggested a significant effect of ratio, $F(1,136)=17.083, p<.001, \mathrm{y}_{\mathrm{p}}{ }^{2}=.112$. The performance in large ratio items was significantly better than in small ratio items (estimated marginal means for large ratio items $=20.927$, for small ratio items $=16.234)$. On the other hand, there was a significant main effect of group, $F(2,136)$ $=4.362, p=.015, \mathrm{y}_{\mathrm{p}}{ }^{2}=.060$. The post hoc analysis suggested that the NA group performed significantly better than the DD group $(p=.012)$, while no other significant group differences were observed $(p s>.07)$. The insignificant Group $\times$ Ratio interaction $(p=.153)$ suggested that the effect of ratio (an indicator of the distance effect) was the same across different groups, which therefore indicated the absence of group difference in distance effect. No other effects were significant ( $p s>.2)$.

It has been suggested that the relation between nonsymbolic comparison task and mathematics achievement was mainly due to the demand of inhibition required in the incongruent trials in the nonsymbolic comparison task (Gilmore et al., 2013). If this hypothesis is true, the performance in the congruent trials should be consistently better than the incongruent trials, and the group differences in nonsymbolic comparison task should be limited to the incongruent trials. However, neither the main effect of congruency, $F(1,272)=.099, p>.7$, nor the Congruency $\times$ Group interaction, $F(2,272)=2.67, p>.07$, reached significance level. This alternative hypothesis was therefore not supported.

For the analysis on $w$, the 3 (Group) $\times 3$ (Time) repeatedmeasures ANCOVA revealed an insignificant effect of time $(p=.158)$. However, there was a main effect of group, $F(2$, $131)=13.230, p<.001, \mathrm{\eta}_{\mathrm{p}}{ }^{2}=.168$. The post hoc analyses suggested that both the NA group and the LA group significantly outperformed the DD group ( $p \mathrm{~s}<.001)$, while the former two groups did not differ significantly $(p=1)$. No other significant main effects or interaction effects were observed $(p s>.2)$.

\section{Dot-Number Matching}

The repeated-measures analysis on dot-number matching accuracy suggested neither significant main effect of time $(p=.790)$ nor significant interaction effects with time $(p s>.5)$. There was a significant main effect of group, 


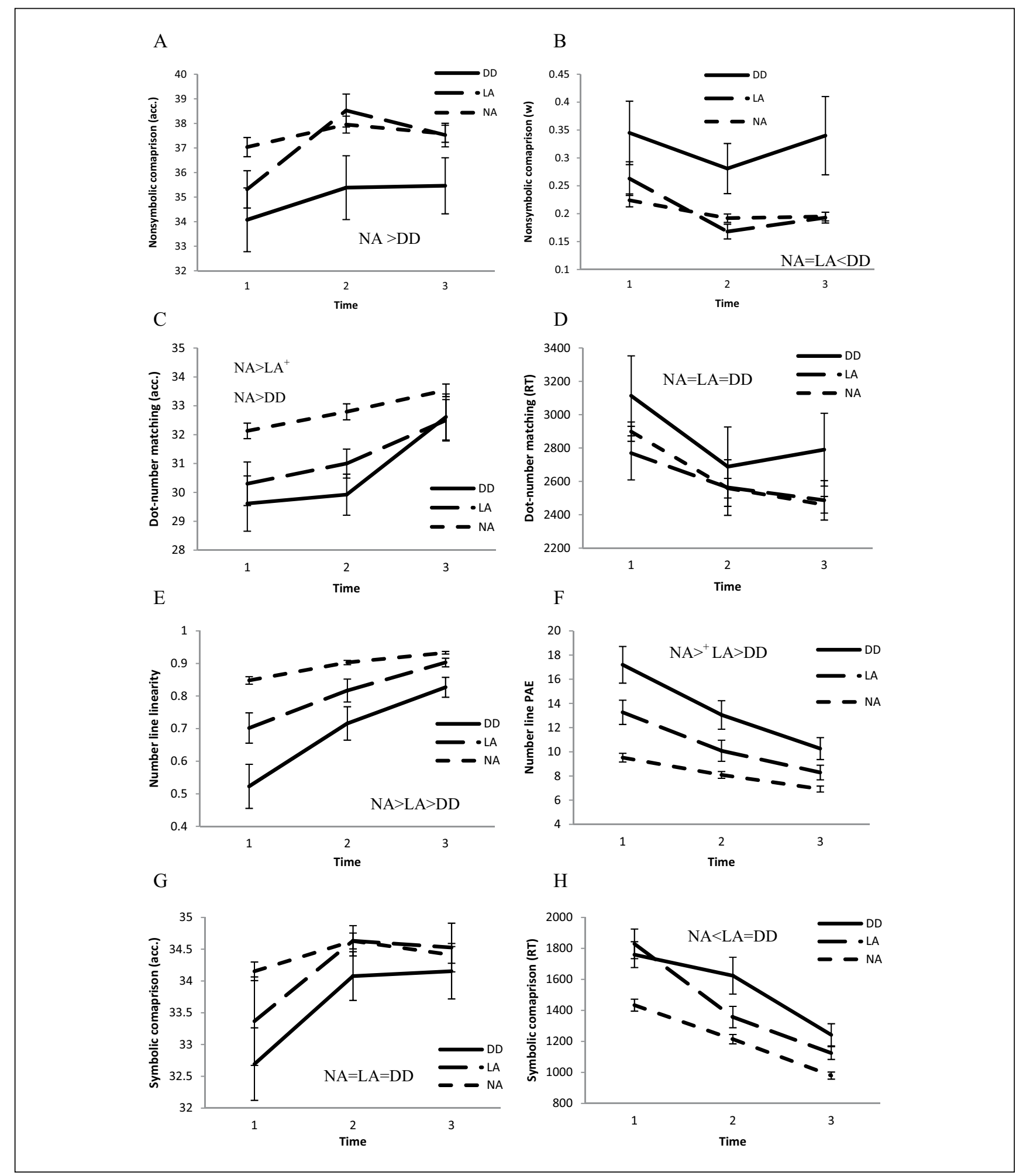

Figure I. Performances of Different Groups in Number-Specific Cognitive Tasks (A: Nonsymbolic Comparison Accuracy; B: Nonsymbolic Comparison w; C: Dot-Number Matching Accuracy; D: Dot-Number Matching Reaction Time; E: Number Line Linearity; F: Number Line Percentage Absolute Error; G: Symbolic Comparison Accuracy; H: Symbolic Comparison Reaction Time) Across Time. Note. Error bars indicate standard errors. ${ }^{+} p<.07$. 
$F(2,134)=4.601, p=.012, \mathrm{y}_{\mathrm{p}}^{2}=.064$. The NA group counted significantly more accurately than the DD group $(p=.036)$ and marginally more accurately then the LA group ( $p=$ .069). The latter two groups did not differ in their counting accuracy $(p=1)$. A similar analysis on the reaction time of the dot-number matching task, however, showed neither significant main effect of time $(p=.184)$, significant main effect of group $(p=.272)$, nor significant interaction effects $(p \mathrm{~s}>.4)$

\section{Number Line}

The repeated-measures ANCOVA on number line linearity suggested a significant main effect of time, $F(1.608$, $207.383)=3.906, p=.030, \mathrm{y}_{\mathrm{p}}{ }^{2}=.029$. The main effect of group was also significant, $F(2,129)=30.525, p<.001$, $\mathrm{y}_{\mathrm{p}}{ }^{2}=.321$. The post hoc analyses suggested that the linearity was highest in the NA group, which was followed by the LA group $(p=.001)$ and then by the DD group ( $p<$ $.001)$. There was also a significant Time $\times$ Group interaction, $F(3.215,207.383)=5.417, p=.001, \mathrm{y}_{\mathrm{p}}{ }^{2}=.077$, suggesting greater improvements among the LA group and the DD group. No other interaction effects reached significant level $(p s>.1)$. The analyses on PAE yielded similar results. There was a significant main effect of group, $F(2$, $133)=13.518, p<.001, \mathrm{y}_{\mathrm{p}}{ }^{2}=.169$. The DD group was significantly outperformed by both the NA group and the LA group $(p \mathrm{~s}<.02)$, while the contrast between the NA group and LA group was marginally significant $(p=.056)$, with the NA group being more accurate. The Time $\times$ Group interaction effect was also significant, $F(3.179,247.305)$ $=4.974, p=.001, \mathrm{y}_{\mathrm{p}}{ }^{2}=.070$, which was driven by the greater improvement among the LA group and the DD group, compared with the NA group. Neither the main effect of time nor its other interactions reached significance level $(p s>.2)$

\section{Symbolic Comparison}

The repeated measures ANCOVA on the accuracy of symbolic comparison task suggested neither the main effect of time $(p=.184)$ nor its interaction effects $(p s>.4)$ were significant. The main effect of group failed to reach significance level $(p=.059)$ after the adjustment of alpha based due to unequal variance, which could be explained by the ceiling effect observed (overall accuracy $>90 \%$ ). The analysis on reaction time of this task yielded a clearer pattern. On top of a significant main effect of time, $F(1.893,249.941)=$ $8.912, p<.001, \mathrm{y}_{\mathrm{p}}{ }^{2}=.063$, a significant main effect of group was also observed, $F(2,132)=7.149, p=.001, \mathrm{y}_{\mathrm{p}}{ }^{2}=$ .098. The NA group significantly outperformed both the LA group $(p=.028)$ and the DD group $(p=.003)$, while the difference between the latter two groups were not significant $(p=.908)$.
To further test whether the deficits in number-magnitude mapping among the DD group was mainly caused by their deficits in the number sense, the group differences between the NA group and the DD group on the number-magnitude mapping variables were further examined using repeated measures ANCOVA, with the average $w$ of the participants across the three time points being controlled. Before $w$ was introduced as a covariate, five out of six of the analyses reached significance level, including dot-number matching accuracy, $F(1,115)=8.123, p=.005, \mathrm{y}_{\mathrm{p}}{ }^{2}=.067$; number line linearity, $F(1,110)=68.585, p<.001, \mathrm{y}_{\mathrm{p}_{2}}^{2}=384$; number line PAE, $F(1,114)=27.828, p<.001, \mathrm{y}_{\mathrm{p}}{ }^{2}=.196$; symbolic comparison accuracy, $F(1,114)=5.568, p=.020$, $\mathrm{y}_{\mathrm{p}}{ }^{2}=.047$; and symbolic comparison reaction time, $F(1$, $114)=10.755, p=.001, \mathrm{j}_{\mathrm{p}}^{2}=.086$. When $w$ was introduced as a covariate in the analyses, its effects were significant in all the analyses $(p s<.05)$. Two out of five significant group differences (dot-number matching accuracy, symbolic comparison accuracy) were eliminated, and one of them became marginally significant (symbolic comparison reaction time, $p=.063)$. Only the group differences in the number line linearity, $F(1,105)=44.055, p<.001, \mathrm{y}_{\mathrm{p}}{ }^{2}=.296$, and number line PAE, $F(1,109)=12.885, p<.001, \mathrm{y}_{\mathrm{p}}{ }^{2}=.106$, remained significant after $w$ was introduced as a covariate (see Table 2 for a summary). The number sense deficits in the DD group seemed to be responsible for at least part of the deficits in number-magnitude mapping in this group.

\section{Discussion}

The current study aimed at examining the relevance of the number sense deficit hypothesis and the access deficit hypothesis in explaining the profile of dyscalculia. Three groups of participants (i.e., DD, LA, and NA) were assessed three times on their number sense and their number-magnitude mapping skills (measured by counting, number line estimation, and symbolic comparison). While the DD group showed difficulties in both number sense and number-magnitude mapping, the LA group showed difficulties only in number-magnitude mapping. Furthermore, the DD group's deficits in number-magnitude mapping were at least partially explained by the number sense deficit. These findings provide support to the number sense deficit hypothesis.

\section{The Core Deficits of Dyscalculia}

In the current literature on dyscalculia, researchers have been debating whether the core deficit among dyscalculics lies in their number sense or the access of magnitude representation from the number symbols. While the number sense deficit hypothesis proposes that the core deficit of dyscalculia lies in the number sense and affects all aspects of numerical processing (Wilson \& Dehaene, 2007), the access deficit hypothesis suggests that the core deficit of 
Table 2. Deficits Experienced by the LA Group and the DD Group.

\begin{tabular}{lccc}
\hline Task & LA & $\begin{array}{c}\text { DD (without } \\
\text { controlling for } w)\end{array}$ & $\begin{array}{c}\text { DD (controlling } \\
\text { for } w)\end{array}$ \\
\hline Nonsymbolic comparison (acc.) & & $\mathrm{X}$ & - \\
Nonsymbolic comparison (w) & & $\mathrm{X}$ & - \\
Nonsymbolic comparison (distance) & $\mathrm{X}^{+}$ & $\mathrm{X}$ & \\
Dot-number matching (acc.) & $\mathrm{X}$ & $\mathrm{X}$ & $\mathrm{X}$ \\
Dot-number matching (RT) & $\mathrm{X}^{+}$ & $\mathrm{X}$ & $\mathrm{X}$ \\
Number line (linearity) & & $\mathrm{X}^{\mathrm{a}}$ & $\mathrm{X}^{+}$ \\
Number line (PAE) & $\mathrm{X}$ & $\mathrm{X}$ & \\
Symbolic comparison (acc.) & & & \\
Symbolic comparison (RT) & & &
\end{tabular}

Note. $\mathrm{LA}=$ low-achieving; $\mathrm{DD}=$ developmental dyscalculia; acc. = accuracy; $w=$ Weber Fraction; $\mathrm{RT}=$ reaction time; $\mathrm{PAE}=$ percentage absolute error. Dashes indicate not applicable.

${ }^{a}$ The contrast between NA and DD was marginally significant when all the three groups were considered, while it was significant when only NA and DD were considered.

${ }^{+} p<.07$.

dyscalculia lies mainly in the access of meaning from number symbols, leaving the nonsymbolic numerosity processing among dyscalculics intact (Rousselle \& Noël, 2007). In the current study, an attempt was made to identify children with DD from a community sample using a more stringent criterion (i.e., bottom 10th percentile in a standardized achievement test). The members of this DD group were outperformed by their NA peers in both indices assessing number sense (nonsymbolic comparison accuracy and $w$ ), hence suggesting a number sense deficit among children with dyscalculia. The above findings converge with those from other studies (e.g., Mazzocco et al., 2011; Piazza et al., 2010) and provide further support to the number sense deficit hypothesis. Furthermore, the present study is the first to report a deficit in the number sense in a much younger DD group (6-7 years old; although the classification was made in Time 4 when the participants were 8 years old, the classification were expected to be largely similar if it were done in other time points given the high stability of children's mathematics achievement, e.g., Aunola, Leskinen, Lerkkanen, \& Nurmi, 2004; Martin et al., 2013; Morgan \& Farkas, 2009), which further suggests that the deficits in number sense among dyscalculics might emerge at an early age. This provides even stronger evidence for the number sense deficit hypothesis. If number sense deficit is the core underlying deficit among dyscalculics, this deficit should be evident early in life.

On top of showing deficit in their number sense, the DD group identified in the present study also showed substandard performance in object counting, estimation, and symbolic comparison tasks. All tasks involve the association between number symbols and the underlying magnitude representation, which provide a reflection of the numbermagnitude mapping process. The substandard performance of the DD group in these tasks therefore suggests that they also suffer from a deficit in their number-magnitude mapping skills. The coexistence of both the number sense deficit and the access deficit among the DD group echoes with the model proposed by the Wong et al. (2015), which suggested a causal role of number sense on number-magnitude mapping. Having an imprecise representation of numerosity may hinder one from associating the number symbols onto the underlying magnitude representation. In other words, a number sense deficit may lead to an access deficit. The findings that the deficits in number-magnitude mapping among the DD group were at least partially explained by their deficits in the number sense provide support to this argument. Future intervention studies can be done to further examine the above proposed mechanism.

It should be noted that, even after controlling for the deficit in the number sense of those with DD, their performance on the number line task was still significantly backward when compared with their NA peers. This means that their substandard performance in the number line task was not completely explained by their number sense deficit. Other domain-general deficits of dyscalculia, such as deficits in spatial processing (Passolunghi \& Mammarella, 2011), may explain the substandard performance in the number line task among this group. This possibility could be further examined in future studies.

\section{Difficulties Experienced by the LA Children}

In addition to the cognitive profiles of the DDs, the cognitive profiles of the LAs are also worth attention. This group of children performed worse than their NA peers in all the number-magnitude mapping tasks, while their performance was comparable to their NA peers in the number sense task. This pattern of results matches well with the access deficit hypothesis. The profiles of the DD group and the LA group 
together seem to suggest that while children with most severe difficulties in learning mathematics suffer from more deep-rooted deficits (i.e., inability in representing numerosity), children with milder forms of the difficulties suffer from deficits that are more peripheral (i.e., associating numbers to the underlying magnitude representation). In a review, Ansari (2008) suggested that the process of mapping the number symbols onto the magnitude representation resembled the mapping between grapheme and phoneme during reading, and both might reflect a domain-general symbols-referent mapping mechanism. It is possible that the LAs are impaired in this domain-general mapping mechanism, which prevents them from accurately associating the number symbols to their corresponding referent and hence results in a difficulty in mathematics learning. If this hypothesis is true, we should also expect a deficit in reading among this group of LA children. Given the high comorbidity between reading and mathematics difficulties (Landerl \& Moll, 2010), the above hypothesis may have some ground. Further studies could be done to verify this.

\section{Addressing the Controversies in the Current Literature}

Findings from the present study also provide some insights to the inconsistent findings in the field of dyscalculia research. First, different studies tended to use different criteria for identifying dyscalculics (see Butterworth, 2005; Murphy et al., 2007, for summaries). While previous studies converged to suggest a prevalence rate of around $6 \%$ to 7\% (Butterworth, 2005; Shalev, 2007), the use of relatively loose criteria in identifying "dyscalculic" children might have resulted in many false positive cases, which might have diluted the group differences in number sense and resulted in insignificant findings. In fact, for those studies that found significant group differences in number sense, most of them used relatively stringent criteria (e.g., Mazzocco et al., 2011; Piazza et al., 2010, and the present study).

Second, the items used in the nonsymbolic comparison task might also contribute to the inconsistent findings in the field. While some studies involved numerosity pairs with larger ratios in the nonsymbolic comparison task (e.g., Iuculano et al., 2008; Rousselle \& Noël, 2007), others involved numerosity pairs with smaller ratios (e.g., Mazzocco et al., 2011; Piazza et al., 2010). Group differences were found only when the nonsymbolic comparison task was sensitive enough (i.e., consisting a considerable portion of items on small ratio numerosity pairs; Mazzocco et al., 2011; Piazza et al., 2010; the present study). In future studies, the nonsymbolic comparison task should be more tailor-made for the particular age group involved in order to increase the sensitivity of measurement.
Third, the use of indices for the nonsymbolic comparison task might also affect the results. While most studies used accuracy, reaction time (e.g., De Smedt \& Gilmore, 2011; Rousselle \& Noël, 2007), and distance effect (e.g., Mussolin, Mejias, \& Noël, 2010) in measuring nonsymbolic numerosity processing, other studies used $w$ to represent one's number acuity (e.g., Mazzocco et al., 2011; Piazza et al., 2010). The present study examined the group differences in accuracy, $w$, and distance effect and found that while there were no group differences found in the distance effect, there were significant group differences with medium to large effect sizes when accuracy and $w$ were used. This may suggest that accuracy and $w$ are more sensitive indices of one's number sense than the distance effect.

\section{Educational Implications}

Besides verifying the number sense deficit hypothesis, the present study also yielded some important practical implications. First, the present study compared the performances of the three groups of children retrospectively since they were in kindergarten. This allows educators to have a better understanding of the profiles of children with low achievement in mathematics even before they went to school. In fact, by comparing the group performance in Time 1 using ANCOVA (with nonverbal intelligence and verbal memory controlled), it was found that the DD group showed some signs of difficulties compared with the control group even before they received formal education on mathematics (nonsymbolic comparison $w: p=.041$; number line linearity and PAE: $p \mathrm{~s}<.001$; symbolic comparison accuracy: $p=$ .066). The findings inform us about the early predictors of dyscalculia, hence making early identification possible. On the other hand, the present findings also shed light on the direction of intervention for the dyscalculics. Given that dyscalculics show deficits in their counting skills, their estimation skills, and their nonsymbolic and symbolic numerical processing, intervention should focus on these domains. Some preliminary success has been observed in terms of interventions for mathematical skills. For example, when training adults on nonsymbolic arithmetic tasks, improvement in their efficiency in solving symbolic arithmetic was observed (Park \& Brannon, 2013). The practice of comparing numerical magnitudes through computer-assisted interventions such as the Number Race (Wilson et al., 2006) and the Graphogame-Math have also been found effective in improving preschoolers' number comparison skills (Räsänen, Salminen, Wilson, Aunio, \& Dehaene, 2009). On the other hand, by regularly playing an interesting number line game, children, regardless of whether they had dyscalculia, improved in their arithmetic skills (Kucian et al., 2011). Furthermore, simply playing linear board games has been found to be helpful to children's math skills (Siegler \& 
Ramani, 2009). All these interventions have provided new hope to educators in helping children with dyscalculia.

\section{Limitations}

The major limitation of the present study was the relatively small sample size of the DD group. This small sample size may limit the generalizability of our findings. However, the patterns revealed by the group comparisons were clear, and the effect sizes were relatively large (all significant comparisons yielded medium or above effect sizes). Furthermore, the findings from the current study converge well with those obtained in other studies (e.g., Mazzocco et al., 2011; Piazza et al., 2010). All these elements suggest that the results obtained in the current study are reliable. By increasing the initial sample size, future studies may yield a larger DD sample for further comparisons.

\section{Conclusions}

The current study compared the dyscalculics with their LA and NA peers in terms of their number sense and numbermagnitude mapping. While the dyscalculics showed deficits in both number sense and number-magnitude mapping, the LA children showed deficits only in number-magnitude mapping. Furthermore, the number-magnitude mapping deficits among the dyscalculics were at least partially explained by their number sense deficit. The findings support the number sense deficit hypothesis. The findings also provide implications for early identification of dyscalculia as well as the design of interventions for these children.

\section{Authors' Note}

Part of the work was done by the first author in the University of Hong Kong.

\section{Declaration of Conflicting Interests}

The author(s) declared no potential conflicts of interest with respect to the research, authorship, and/or publication of this article.

\section{Funding}

The author(s) received no financial support for the research, authorship, and/or publication of this article.

\section{References}

Ansari, D. (2008). Effects of development and enculturation on number representation in the brain. Nature Reviews Neuroscience, 9(4), 278-291. doi: 10.1038/nrn2334

Aunola, K., Leskinen, E., Lerkkanen, M.-K., \& Nurmi, J.-E. (2004). Developmental dynamics of math performance from preschool to grade 2. Journal of Educational Psychology, 96(4), 699-713. doi: 10.1037/0022-0663.96.4.699
Booth, J. L., \& Siegler, R. S. (2006). Developmental and individual differences in pure numerical estimation. Developmental Psychology, 42(1), 189-201. doi: 10.1037/0012-1649.41.6.189

Butterworth, B. (2003). Dyscalculia screener. Computer. London, UK: nfer Nelson.

Butterworth, B. (2005). Developmental dyscalculia. In J. I. D. Campbell (Ed.), Handbook of mathematical cognition (pp. 455-468). New York, NY: Psychology Press.

Carey, S. (2004). Bootstrapping \& the origin of concepts. Daedalus, 133, 59-68. doi: 10.1162/001152604772746701

Chen, Q., \& Li, J. (2014). Association between individual differences in non-symbolic number acuity and math performance: A meta-analysis. Acta Psychologica, 148, 163-172. doi: 10.1016/j.actpsy.2014.01.016

De Smedt, B., \& Gilmore, C. K. (2011). Defective number module or impaired access? Numerical magnitude processing in first graders with mathematical difficulties. Journal of Experimental Child Psychology, 108(2), 278-292. doi: 10.1016/j.jecp.2010.09.003

De Smedt, B., Noël, M.-P., Gilmore, C., \& Ansari, D. (2013). How do symbolic and non-symbolic numerical magnitude processing skills relate to individual differences in children's mathematical skills? A review of evidence from brain and behavior. Trends in Neuroscience and Education, 2(2), 4855. doi: 10.1016/j.tine.2013.06.001

Dehaene, S. (2001). Précis of "The number sense." Mind and Language, 16(1), 16-36. doi: 10.1111/1468-0017.00154

Dehaene, S. (2005). Evolution of human cortical circuits for reading and arithmetic: The "neuronal recycling" hypothesis . In S. Dehaene, J. R. Duhamel, M. Hauser, \& G. Rizzolatti (Eds.), From monkey brain to human brain (pp. 133-157). Cambridge, MA: MIT Press.

Dehaene, S., Piazza, M., Pinel, P., \& Cohen, L. (2003). Three parietal circuits for number processing. Cognitive Neuropsychology, 20(3), 487-506. doi: 10.1080/02643290244000239

Feigenson, L., Dehaene, S., \& Spelke, E. (2004). Core systems of number. Trends in Cognitive Sciences, 8(7), 307-314. doi: 10.1016/j.tics.2004.05.002

Fuchs, L. S., Geary, D. C., Compton, D. L., Fuchs, D., Hamlett, C. L., Seethaler, P. M., \& Schatschneider, C. (2010). Do different types of school mathematics development depend on different constellations of numerical versus general cognitive abilities? Developmental Psychology, 46(6), 1731-1746. doi: 10.1037/a0020662

Geary, D. C., Hamson, C. O., \& Hoard, M. K. (2000). Numerical and arithmetical cognition: A longitudinal study of process and concept deficits in children with learning disability. Journal of Experimental Child Psychology, 77(3), 236-263. doi: 10.1006/jecp.2000.2561

Geary, D. C., Hoard, M. K., Byrd-Craven, J., \& Nugent, L. (2007). Cognitive mechanisms underlying achievement deficits in children with mathematical learning disability. Child Development, 78(4), 1343-1359. doi: 10.1111/j.14678624.2007.01069.x

Geary, D. C., Hoard, M. K., Nugent, L., \& Byrd-Craven, J. (2008). Development of number line representations in children with mathematical learning disability. Developmental Neuropsychology, 33(3), 277-299. doi: 10.1080/ 87565640801982361 
Gilmore, C., Attridge, N., Clayton, S., Cragg, L., Johnson, S., Marlow, N., \& Inglis, M. (2013). Individual differences in inhibitory control, not non-verbal number acuity, correlate with mathematics achievement. PloS One, 8(6), e67374. doi: 10.1371/journal.pone.0067374

Halberda, J., Ly, R., Wilmer, J. B., Naiman, D. Q., \& Germine, L. (2012). Number sense across the lifespan as revealed by a massive Internet-based sample. Proceedings of the National Academy of Sciences of the United States of America, 109(28), 11116-11120. doi: 10.1073/pnas.1200196109

Halberda, J., Mazzocco, M. M. M., \& Feigenson, L. (2008). Individual differences in non-verbal number acuity correlate with maths achievement. Nature, 455, 665-668. doi: 10.1038/ nature 07246

Hanich, L. B., Jordan, N. C., Kaplan, D., \& Dick, J. (2001). Performance across different areas of mathematical cognition in children with learning difficulties. Journal of Educational Psychology, 93, 615-626. doi: 10.1037/0022-0663.93.3.615

Hong Kong Education Bureau. (2008). The learning and achievement measurement kit. Hong Kong: Hong Kong SAR Government.

Iuculano, T., Tang, J., Hall, C. W. B., \& Butterworth, B. (2008). Core information processing deficits in developmental dyscalculia and low numeracy. Developmental Science, 11(5), 669680. doi: 10.1111/j.1467-7687.2008.00716.x

Kucian, K., Grond, U., Rotzer, S., Henzi, B., Schönmann, C., Plangger, F., \& von Aster, M. (2011). Mental number line training in children with developmental dyscalculia. NeuroImage, 57(3), 782-795. doi: 10.1016/j.neuroimage.2011.01.070

Landerl, K. (2013). Development of numerical processing in children with typical and dyscalculic arithmetic skills-a longitudinal study. Frontiers in Psychology, 4, 459. doi: 10.3389/ fpsyg.2013.00459

Landerl, K., Bevan, A., \& Butterworth, B. (2004). Developmental dyscalculia and basic numerical capacities: A study of 8-9-year-old students. Cognition, 93(2), 99-125. doi: 10.1016/j.cognition.2003.11.004

Landerl, K., \& Kölle, C. (2009). Typical and atypical development of basic numerical skills in elementary school. Journal of Experimental Child Psychology, 103(4), 546-565. doi: 10.1016/j.jecp.2008.12.006

Landerl, K., \& Moll, K. (2010). Comorbidity of learning disorders: Prevalence and familial transmission. Journal of Child Psychology and Psychiatry, 51(3), 287-294. doi: 10.1111/j.1469-7610.2009.02164.x

Libertus, M. E., Feigenson, L., \& Halberda, J. (2013). Is approximate number precision a stable predictor of math ability? Learning and Individual Differences, 25, 126-133. doi: 10.1016/j.lindif.2013.02.001

Martin, R. B., Cirino, P. T., Barnes, M. A., Ewing-Cobbs, L., Fuchs, L. S., Stuebing, K. K., \& Fletcher, J. M. (2013). Prediction and stability of mathematics skill and difficulty. Journal of Learning Disabilities, 46(5), 428-443. doi: 10.1177/0022219411436214

Mazzocco, M. M. M., Feigenson, L., \& Halberda, J. (2011). Impaired acuity of the approximate number system underlies mathematical learning disability (dyscalculia). Child Development, 82(4), 1224-1237. doi: 10.1111/j.14678624.2011.01608.x
Morgan, P. L., \& Farkas, G. (2009). Five-year growth trajectories of kindergarten children with learning difficulties in mathematics. Journal of Learning Disabilities, 42(4), 306-321. doi: $10.1177 / 0022219408331037$

Murphy, M. M., Mazzocco, M. M. M., Hanich, L. B., \& Early, M. C. (2007). Cognitive characteristics of children with mathematics learning disability (MLD) vary as a function of the cutoff criterion used to define MLD. Journal of Learning Disabilities, 40(5), 458-478. doi: 10.1177/00222194070400050901

Mussolin, C., Mejias, S., \& Noël, M.-P. (2010). Symbolic and nonsymbolic number comparison in children with and without dyscalculia. Cognition, 115(1), 10-25. doi: 10.1016/j.cognition.2009.10.006

Noël, M.-P., \& Rousselle, L. (2011). Developmental changes in the profiles of dyscalculia: An explanation based on a double exact-and-approximate number representation model. Frontiers in Human Neuroscience, 5, 165. doi: 10.3389/ fnhum.2011.00165

Park, J., \& Brannon, E. (2013). Training the approximate number system improves math proficiency. Psychological Science, 24(10), 2013-2019. doi: 10.1177/0956797613482944

Parsons, S., \& Bynner, J. (2005). Does numeracy matter more? London, UK: National Research and Development Centre for Adult Literacy and Numeracy.

Passolunghi, M. C., \& Mammarella, I. C. (2011). Selective spatial working memory impairment in a group of children with mathematics learning disabilities and poor problem-solving skills. Journal of Learning Disabilities, 45(4), 341-50. doi: 10.1177/0022219411400746

Piazza, M., Facoetti, A., Trussardi, A. N., Berteletti, I., Conte, S., Lucangeli, D., \& Zorzi, M. (2010). Developmental trajectory of number acuity reveals a severe impairment in developmental dyscalculia. Cognition, 116(1), 33-41. doi: 10.1016/j.cognition.2010.03.012

Piazza, M., Izard, V., Pinel, P., Le Bihan, D., \& Dehaene, S. (2004). Tuning curves for approximate numerosity in the human intraparietal sulcus. Neuron, 44(3), 547-555. doi: 10.1016/j.neuron.2004.10.014

Räsänen, P., Salminen, J., Wilson, A. J., Aunio, P., \& Dehaene, S. (2009). Computer-assisted intervention for children with low numeracy skills. Cognitive Development, 24(4), 450-472. doi: 10.1016/j.cogdev.2009.09.003

Raven, J. C. (1956). Standard progressive matrices sets $A, B, C, D$ \& E. Oxford, UK: Psychologists Press.

Reeve, R., Reynolds, F., Humberstone, J., \& Butterworth, B. (2012). Stability and change in markers of core numerical competencies. Journal of Experimental Psychology. General, 141(4), 649-666. doi: 10.1037/a0027520

Reigosa-Crespo, V., Valdés-Sosa, M., Butterworth, B., Estévez, N., Rodríguez, M., Santos, E., \& Lage, A. (2012). Basic numerical capacities and prevalence of developmental dyscalculia: The Havana Survey. Developmental Psychology, 48(1), 123-135. doi: 10.1037/a0025356

Rousselle, L., \& Noël, M.-P. (2007). Basic numerical skills in children with mathematics learning disabilities: A comparison of symbolic vs non-symbolic number magnitude processing. Cognition, 102(3), 361-395. doi: 10.1016/j.cognition.2006.01.005 
Shalev, R. S. (2007). Prevalence of developmental dyscalculia. In D. B. Berch \& M. M. M. Mazzocco (Eds.), Why is math so hard for some children? The nature and origins of mathematical learning difficulties and disabilities (pp. 49-60). Baltimore, MD: Paul H. Brookes.

Siegler, R. S., \& Booth, J. L. (2004). Development of numerical estimation in young children. Child Development, 75(2), 428 444. doi: 10.1111/j.1467-8624.2004.00684.x

Siegler, R. S., \& Ramani, G. B. (2009). Playing linear number board games-but not circular ones-improves lowincome preschoolers' numerical understanding. Journal of Educational Psychology, 101(3), 545-560. doi: 10.1037/ a0014239

Starr, A., Libertus, M. E., \& Brannon, E. M. (2013). Number sense in infancy predicts mathematical abilities in childhood. Proceedings of the National Academy of Sciences of the United States of America, 110(45), 18116-18120. doi: 10.1073/pnas. 1302751110

Tabachnick, B., \& Fidell, L. (2006). Using multivariate statistics (5th ed.). Needham Heights, MA: Allyn \& Bacon.

Wilson, A. J., \& Dehaene, S. (2007). Number sense and developmental dyscalculia. In D. Coch, G. Dawson, \& K. W. Fischer (Eds.), Human behavior, learning, and the developing brain: Atypical development (pp. 212-238). New York, NY: Guilford Press.

Wilson, A. J., Dehaene, S., Pinel, P., Revkin, S. K., Cohen, L., \& Cohen, D. (2006). Principles underlying the design of "The Number Race", an adaptive computer game for remediation of dyscalculia. Behavioral and Brain Functions: BBF, 2, 19. doi: 10.1186/1744-9081-2-19

Wong, T. T.-Y., Ho, C. S.-H., \& Tang, J. (2015). The relation between ANS and arithmetic achievement: The mediating role of numbernumerosity mapping. Manuscript submitted for publication. 